\begin{tabular}{ll}
\hline \hline MINING AND METALLURGY INSTITUTE BOR & ISSN: 2334-8836 \\
& UDK: 622 \\
\hline \hline
\end{tabular}

UDK: $622.841: 504.06(045)=111$

DOI:10.5937/MMEB1403033M

Vladan Marinković, Ljubiša Obradović, Mile Bugarin", Goran Stojanović

\title{
THE IMPACT OF POLLUTED WASTEWATER ON WATER QUALITY OF THE BOR RIVER AND SURROUNDING GROUNDWATER ${ }^{* * *}$
}

\begin{abstract}
Due to the immediate vicinity of the mining and industrial facilities, the water pollution in the Bor River is extremely high. Sources of the Bor River pollution are active and inactive mining operations (surface and underground), flotation tailing dumps, waste rock dumps from the open pit, waste water generated in the process of copper ore processing and municipal wastewater.

The Bor River basin has an area of approximately $61.0 \mathrm{~km}^{2}$. Maximum length of the river flow is about $10.0 \mathrm{~km}$ with an altitude difference of $160 \mathrm{~m}$. The Bor River belongs to the basin of the Bela River, the basin of the River Timok or the Danube basin. In its course, it runs through the village of Slatina, connecting downstream of the village with the Krivelj River and Ravna River forming the Bela River. The Bela River, downstream from the village of Vražogrnac flows into the River Veliki Timok, which empties into the Danube [1] [2].

Wastewater from the mining and industrial facilities are still discharged directly into the river, so the water pollution in the river has also a large impact on the ground water pollution, especially in the area of the village of Slatina, located downstream from the town of Bor.

In addition to the waste water, discharged into the Bor River, the major threat to the environment is also a large amount of flotation tailings, located on the coast of this river, which, due to the frequent accidents, occurred in the Bor Flotation Plant during the last century, was directly discharged into the Bor River [3].

This paper work gives a detailed overview of the quality of surface and groundwater both of the Bor River and water polluting of this river.
\end{abstract}

Keywords: Bor River, waste water, underground water, pollution

\section{INTRODUCTION}

Due to the immediate vicinity of the mining and industrial facilities, the water pollution in the Bor River is extremely high. Sources of the Bor River pollution are active and inactive mining operations

(surface and underground), flotation tailing dumps, waste rock dumps from the open pit, waste water generated in the process of copper ore processing and municipal wastewater (Figure 1) [4] [5].

\footnotetext{
* Mining and Metallurgy Institute Bor

** This work is the result of the Project TR37001 "The Impact of Mining Waste from RTB Bor on the Pollution of Surrounding Water Systems with the Proposal of Measures and Procedures for Reduction Harmful Effects on the Environment", funded by the Ministry of Education, Science and Technological Development of the Republic of Serbia
} 


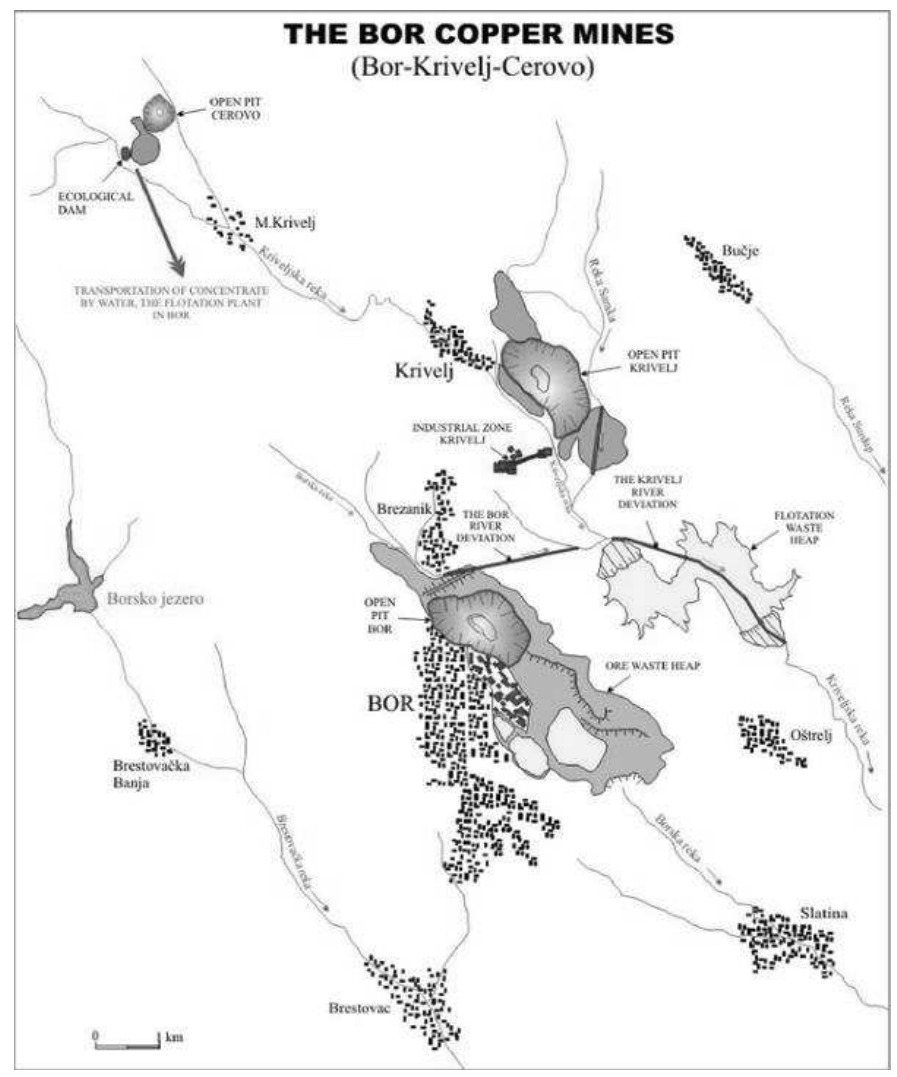

Figure 1 Waste water generated in RBB Bor and TIR complex

In addition to the waste water, discharged into the Bor River, the major threat to the environment is also a large amount of flotation tailings, located on the coast of this river, which, due to the frequent accidents, occurred in the Bor Flotation Plant during the last century, was directly discharged into the Bor River [3].

\section{THE IMPACT OF POLLUTED WASTEWATER}

The Bor River basin has an area of approximately $61.0 \mathrm{~km}^{2}$. Maximum length of the river flow is about $10.0 \mathrm{~km}$ with an altitude difference of $160 \mathrm{~m}$. The Bor River belongs to the basin of the Bela River, the basin of the River Timok or the Danube basin. In its course, it runs through the village of Slatina, connecting downstream of the village with the Krivelj River and Ravna River forming the Bela River. The Bela River, downstream from the village of Vražogrnac flows into the River Veliki Timok, which empties into the Danube [1].

Waste water from mining and industrial facilities is still discharged directly into the river, so the water pollution in the river has also a large impact on the ground water pollution, especially in the area of the village of Slatina, located downstream from the town of Bor [4] [5], (Figure 1).

Such pollution has a very large negative impact on the health of residents of the village of Slatina since the coastal of the Bor River is used for different purposes [4] [5], (Figure 2). 


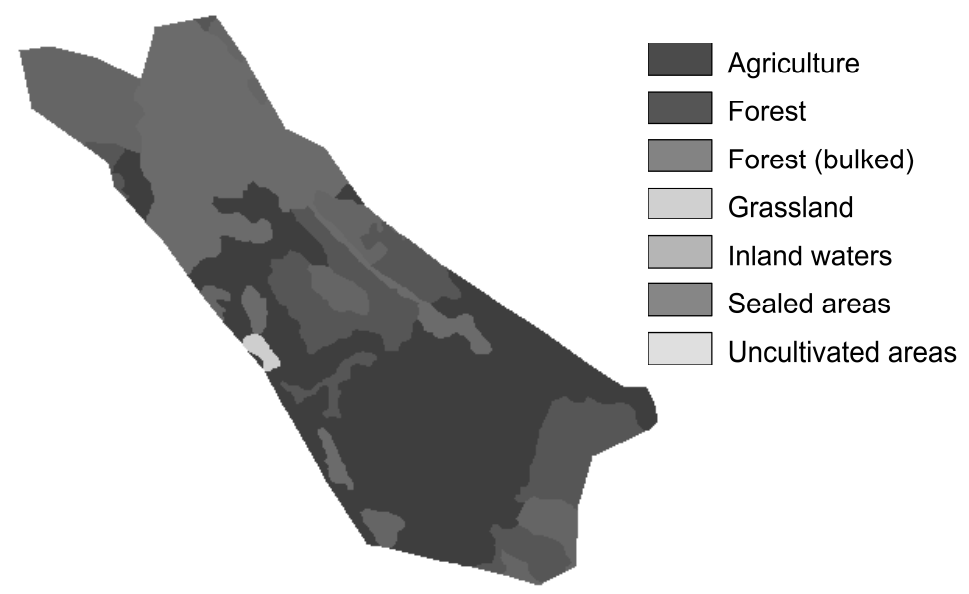

Figure 2 The use of land in the catchment area of the Bor River

Proportions of the land use are listed in Table 1.

Table 1 Proportions of the catchment area of the Bor River

\begin{tabular}{|l|l|}
\hline \multicolumn{2}{|c|}{ Land use [\%] } \\
\hline Cereals, fodder plants & Cereals, fodder plants \\
\hline Grassland & Grassland \\
\hline Forest (very bulked) & Forest (very bulked) \\
\hline Forest (medium) & Forest (medium) \\
\hline Sealed areas & Sealed areas \\
\hline
\end{tabular}

In the period from February to June out twice a month. Tables 2 and 2 a present 2011, a continuous sampling of water some of the results of water analyses from samples from the Bor River was carried the Bor River.

Tables 2 and 2a Results of physical-chemical testing the samples from the location of the Bor River

\begin{tabular}{|c|c|c|c|c|c|c|c|c|}
\hline $\begin{array}{c}\text { Sampling } \\
\text { spot }\end{array}$ & $\begin{array}{c}\mathbf{T}\left({ }^{0} \mathbf{C}\right) \\
\text { air }\end{array}$ & $\begin{array}{c}\mathbf{T}\left({ }^{0} \mathbf{C}\right) \\
\text { water }\end{array}$ & $\begin{array}{c}\text { Color/ } \\
\text { smell }\end{array}$ & $\begin{array}{c}\text { Electrical } \\
\text { conductivity } \\
\mathbf{\mu S} / \mathbf{c m}\end{array}$ & $\mathbf{p H}$ & $\begin{array}{c}\mathbf{C u} \\
\mathbf{m g} / \mathbf{d m}^{\mathbf{3}}\end{array}$ & $\begin{array}{c}\mathbf{P b} \\
\mathbf{m g} / \mathbf{d m}^{\mathbf{3}}\end{array}$ & $\begin{array}{c}\mathbf{Z n} \\
\mathbf{m g} / \mathbf{d m}^{\mathbf{3}}\end{array}$ \\
\hline Bor River & 17.2 & 17.6 & $\begin{array}{c}\text { muddy/ } \\
\text { without }\end{array}$ & 1472 & 5.00 & 16.9 & 0.23 & 2.9 \\
\hline
\end{tabular}

\begin{tabular}{|c|c|c|c|c|c|c|c|c|}
\hline $\begin{array}{c}\text { Sampling } \\
\text { spot }\end{array}$ & $\begin{array}{c}\mathbf{C d} \\
\left(\mathbf{m g} / \mathbf{d m}^{\mathbf{3}}\right)\end{array}$ & $\begin{array}{c}\mathbf{N i} \\
\left(\mathbf{m g} / \mathbf{d m}^{\mathbf{3}}\right)\end{array}$ & $\begin{array}{c}\mathbf{C r} \\
\left(\mathbf{m g} / \mathbf{d m}^{\mathbf{3}}\right)\end{array}$ & $\begin{array}{c}\text { Se } \\
\left(\mathbf{m g} / \mathbf{d m}^{\mathbf{3}}\right)\end{array}$ & $\begin{array}{c}\mathbf{A s} \\
\left(\mathbf{m g} / \mathbf{d m}^{\mathbf{3}}\right)\end{array}$ & $\begin{array}{c}\mathbf{F e}-t o t a l \\
\left(\mathbf{m g} / \mathbf{d m}^{\mathbf{3}}\right)\end{array}$ & $\begin{array}{c}\text { Susp.matters } \\
\left(\mathbf{m g} / \mathbf{d m}^{\mathbf{3}}\right)\end{array}$ & $\begin{array}{c}\mathbf{S O}_{\mathbf{4}}{ }^{-2} \\
\left(\mathbf{m g} / \mathbf{d m}^{\mathbf{3}}\right)\end{array}$ \\
\hline Bor River & $<0.1$ & 0.37 & $<0.1$ & $<0.2$ & $<0.1$ & 36.9 & 899.0 & 1204.3 \\
\hline
\end{tabular}

Testing Also, in the same period, water samples were taken twice a month from piezometers in the coastal of the Bor $\mathrm{Ri}$ - ver. Tables 3 and $3 a$ present some of the results of water analyses from this piezometer. 
Tables 3 and 3a Results of physical-chemical testing the samples of ground water

\begin{tabular}{|c|c|c|c|c|c|c|c|c|}
\hline $\begin{array}{c}\text { Sampling } \\
\text { Spot }\end{array}$ & $\begin{array}{c}\left.\text { T ( }{ }^{\mathbf{0}} \mathbf{C}\right) \\
\text { air }\end{array}$ & $\begin{array}{c}\left.\text { T ( }{ }^{\mathbf{0}} \mathbf{C}\right) \\
\text { water }\end{array}$ & Color/smell & $\begin{array}{c}\text { Electrical } \\
\text { conductivity } \\
\boldsymbol{\mu S} \mathbf{S} / \mathbf{c m}\end{array}$ & $\mathbf{p H}$ & $\begin{array}{c}\mathbf{C u} \\
\mathbf{m g} / \mathbf{d m}^{\mathbf{3}}\end{array}$ & $\begin{array}{c}\mathbf{P b} \\
\mathbf{m g} / \mathbf{d m}^{\mathbf{3}}\end{array}$ & $\begin{array}{c}\mathbf{Z n} \\
\mathbf{m g} / \mathbf{d m}^{\mathbf{3}}\end{array}$ \\
\hline P4 & 20 & 16.0 & $\begin{array}{c}\text { muddy/ } \\
\text { without }\end{array}$ & 1777 & 5.21 & 5.1 & $<0.1$ & 2.9 \\
\hline
\end{tabular}

\begin{tabular}{|c|c|c|c|c|c|c|c|c|}
\hline $\begin{array}{c}\text { Sampling } \\
\text { spot }\end{array}$ & $\begin{array}{c}\mathbf{C d} \\
\left(\mathbf{m g} / \mathbf{d m}^{\mathbf{3}}\right)\end{array}$ & $\begin{array}{c}\mathbf{~ N i} \\
\left(\mathbf{m g} / \mathbf{d m}^{\mathbf{3}}\right)\end{array}$ & $\begin{array}{c}\mathbf{C r} \\
\left(\mathbf{m g} / \mathbf{d m}^{\mathbf{3}}\right)\end{array}$ & $\begin{array}{c}\text { Se } \\
\left(\mathbf{m g} / \mathbf{d m}^{\mathbf{3}}\right)\end{array}$ & $\begin{array}{c}\mathbf{A s} \\
\left(\mathbf{m g} / \mathbf{d m}^{\mathbf{3}}\right)\end{array}$ & $\begin{array}{c}\text { Fe-total } \\
\left(\mathbf{m g} / \mathbf{d m}^{\mathbf{3}}\right)\end{array}$ & $\begin{array}{c}\text { Susp.matters } \\
\left(\mathbf{m g} / \mathbf{d m}^{\mathbf{3}}\right)\end{array}$ & $\begin{array}{c}\mathbf{S O}_{\mathbf{4}}{ }^{-2} \\
\left(\mathbf{m g} / \mathbf{d m}^{\mathbf{3}}\right)\end{array}$ \\
\hline $\mathrm{P} 4$ & $<0.1$ & 0.37 & $<0.1$ & $<0.2$ & $<0.1$ & 36.9 & 899.0 & 1204.3 \\
\hline
\end{tabular}

\section{CONCLUSION}

In the Republic of Serbia, the protection of surface water is according to the legislation which made the water classification into four classes according to the level of pollution and use. The limit values of elements are given in the Official Gazette of the Republic of Serbia No. 31/82 (Chemical Quality Parameters).

Surface water within the Bor River cannot be classified (according to their composition) neither in the class IV and they fall into the water with high levels of potentially toxic elements (heavy metals) according to the level of maximum allowable concentration.

Such pollution has a very large negative impact on the health of residents of the village of Slatina, located downstream from the town of Bor and industrial complexes which pollute the Bor River, and which flows through the village itself.

The favorable copper prices on the world market have also affected the copper production in Bor that grows from year to year. Also, the pollution level in the Bor River increases with increased production. In order to reduce the level of pollution in the Bor River, it is necessary as soon as possible to access the modern methods of remediation both of polluted industrial water and coastal contaminated land.

\section{REFERENCES}

[1] K. Marika, R. Cupac, B. Vulikić, D. Simić, A. Kodzoman, A. Nacev, E. Jusufi, I. Derguti, S. Dragojević, S. Marstijepović, 2007-2010, Program Western Balkans Environmental Programme Final Report (UNDP);

[2] Group of authors, 2002, Assessment of Environmental Monitoring Capacities in Bor, (UNEP);

[3] Group of authors, 2007, RTB Bor Tailing Ponds Remediation Study Final Report;

[4] Group of authors, 2011, Feasibility Study for Remediation the Ground and Surface Water in the Bor Mine;

[5] Intreat - FP6 The Project funded by the EU - Integrated Waste Treatment in Order to Prevent Pollution of the Danube River, MMI was the partner in the Project, Project Implemented from July 2004 to August 2007;

[6] Regulation of the Republic of Serbia on the Landfills ("Official Gazette" RS No. 92/2010).

[7] D. Ignjatović, L. Djurdjevac Ignjatović, M. Ljubojev: Effect of Displacement the Flotation Dam 2 on the Route of Future Collector of the Krivelj River, Tested Using the Software Phase2 v8.0, Mining and Metallurgy Engineering Bor, 4/2013, pp. 17-22.

[8] Lj. Obradović, J. Stanković, M. Bugarin: Disposal of Hazardous Mining Waste - the Current Serbian and EU Legislation, Mining and Metallurgy Engineering Bor, 3/2013, pp. 113-118. 


\begin{tabular}{ll}
\hline \hline INSTITUT ZA RUDARSTVO I METALURGIJU BOR & ISSN: 2334-8836 \\
& UDK: 622 \\
\hline \hline
\end{tabular}

Vladan Marinkovic ${ }^{*}$, Ljubisa Obradović, Mile Bugarin", Goran Stojanović

\section{UTICAJ ZAGAĐENIH OTPADNIH VODA NA KVALITET VODE BORSKE REKE I OKOLNIH PODZEMNIH VODA**}

\section{Izvod}

Usled neposredne blizine rudničkih i industrijskih objekata zagađenost vode u Borskoj reci je izuzetno visoka. Izvore zagađenja Borske reke predstavljaju aktivni $i$ neaktivni rudarski radovi (površinski i podzemni), flotacijska jalovišta, odlagališta kopovske jalovine, otpadne vode nastale u procesu prerade rude bakra i komunalne otpadne vode.

Sliv Borske reke je površine oko 61,0 km². Maksimalna dužina toka je oko $10.0 \mathrm{~km}$ sa visinskom razlikom od 160 m. Borska reka pripada slivu Bele Reka, slivu reke Timok, odnosno slivu Dunava. U svom toku protiče kroz selo Slatinu, spajajući se nizvodno od sela sa Kriveljskom rekom i Ravnom rekon formirajući Belu reku. Bela reka se nizvodno od sela Vražogrnac uliva u reku Veliki Timok, koji se uliva u reku Dunav [1] [2].

Otpadne vode iz rudničkih i industrijskih objekata se još uvek ispuštaju direktno u reku, tako da zagađenje vode u reci ima velikog uticaja $i$ na zagađenje podzemnih voda, pogotovo u oblasti sela Slatina koje se nalazi nizvodno od grada Bora.

Pored otpadnih voda koje se ispuštaju u Borsku reku veliku opasnost po životnu sredinu predstavlja $i$ velika količina flotacijske jalovine koja se nalazi u priobalju ove reke, koja je usled čestih akcidentnih situacija koja su se dešavala u Borskoj flotaciji tokom prošlog veka ispuštana direktno u Borsku reku [3].

Ovaj rad daje detaljan prikaz kvaliteta površinskih i podzemnih voda Borske reke i voda koje ovu reku zagađuju.

Ključne reči: Borska reka, otpadne vode, podzemne vode, zagađenje

\section{UVOD}

Usled neposredne blizine rudničkih i industrijskih objekata zagađenost vode $\mathrm{u}$ Borskoj reci je izuzetno visoka. Izvore zagađenja Borske reke predstavljaju aktivni i neaktivni rudarski radovi (povr- šinski i podzemni), flotacijska jalovišta, odlagališta kopovske jalovine, otpadne vode nastale $\mathrm{u}$ procesu prerade rude bakra i komunalne otpadne vode (slika 1) [4] [5].

\footnotetext{
* Institut za rudarstvo i metalurgiju Bor

** Ovaj rad je proistekao iz projekta TR37001 „Uticaj rudarskog otpada iz RTB Bor na zagađenje vodotokova, sa predlogom mera i postupaka za smanjenje štetnog dejstva na životnu sredinu “, koji je finansiran sredstvima Ministarstva prosvete, nauke i tehnološkog razvoja Republike Srbije
} 


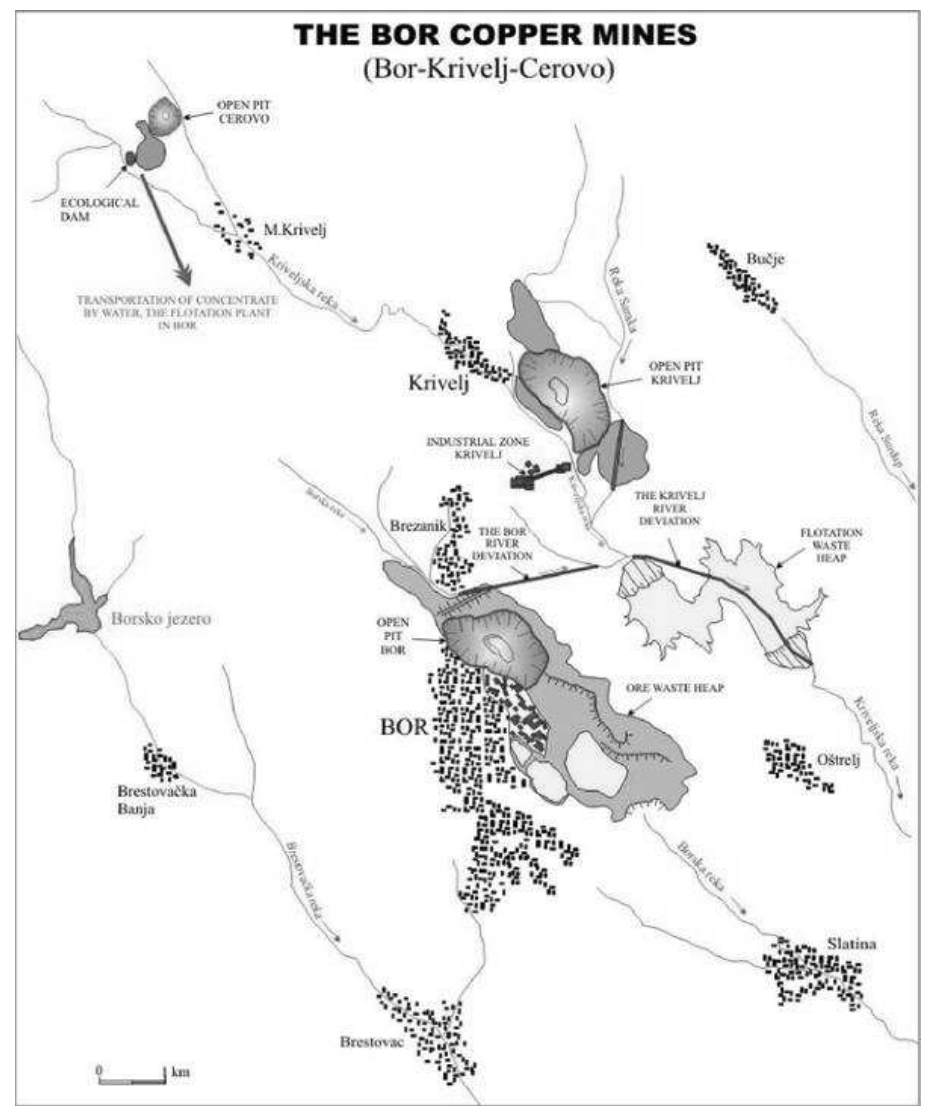

SI. 1. Otpadne vode nastale u RBB Bor i TIR kompleksu

Pored otpadnih voda koje se ispuštaju u Borsku reku veliku opasnost po životnu sredinu predstavlja i velika količina flotacijske jalovine koja se nalazi u priobalju ove reke, koja je usled čestih akcidentnih situacija koja su se dešavala u Borskoj flotaciji tokom prošlog veka ispuštana direktno u Borsku reku [3].

\section{UTICAJ ZAGAĐENIH OTPADNIH VODA}

Sliv Borske reke je površine oko 61,0 $\mathrm{km}^{2}$. Maksimalna dužina toka je oko 10.0 $\mathrm{km}$ sa visinskom razlikom od $160 \mathrm{~m}$. Borska reka pripada slivu Bele Reka, slivu reke Timok, odnosno slivu Dunava. U svom toku protiče kroz selo Slatinu, spajajući se nizvodno od sela sa Kriveljskom rekom i Ravnom rekon formirajući Belu reku. Bela reka se nizvodno od sela Vražogrnac uliva u reku Veliki Timok, koji se uliva u reku Dunav [1].

Otpadne vode iz rudničkih i industrijskih objekata se još uvek ispuštaju direktno u reku, tako da zagađenje vode u reci ima velikog uticaja i na zagađenje podzemnih voda, pogotovo u oblasti sela Slatina koje se nalazi nizvodno od grada Bora [4] [5], (slika 1).

Ovakvo zagađenje ima izuzetno veliki negativan uticaj na zdravlje stanovnika sela Slatina obzirom da se priobalje Borske reke koristi u različite namene [4] [5], (slika 2). 


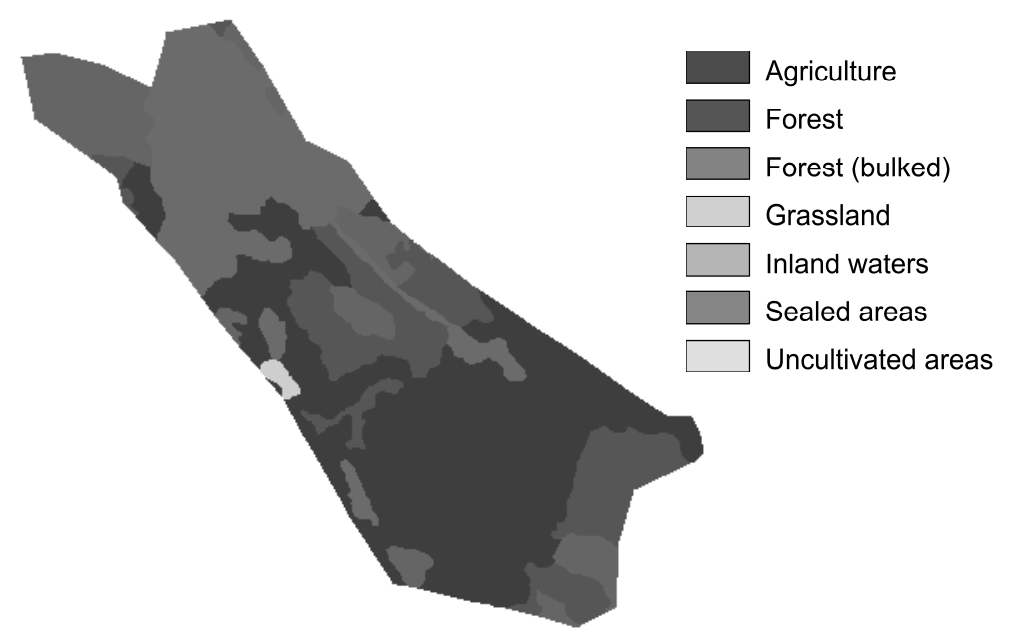

S1. 2. Upotreba zemljišta u slivu Borske reke

Procentualni udeo pojedinih vrsta navedeni su u tabeli 1. upotrebe zemljišta u slivu Borske reke

Tabela 1. Proporcija u slivu Borske reke

\begin{tabular}{|l|r|}
\hline \multicolumn{2}{|c|}{ Upotreba zemljišta [\%] } \\
\hline Žitarice, krmno bilje & 43,83 \\
\hline Livade & 1,70 \\
\hline Šume (vrlo prostrane) & 12,77 \\
\hline Šume (srednje) & 19,15 \\
\hline Zatvorene oblasti & 22,55 \\
\hline
\end{tabular}

U periodu od februara do juna meseca uzorkovanje je vršeno dva puta mesečno. 2011. godine je vršeno kontininualno U tabelama 2 i 2 a su prikazani neki od uzimannje uzoraka vode iz Borske reke, rezultata analiza vode iz Borske reke.

Tabela 2 i 2a. Rezultati fizičko hemijskih ispitivanja uzoraka vode iz Borske reke

\begin{tabular}{|c|c|c|c|c|c|c|c|c|}
\hline $\begin{array}{c}\text { Mesto } \\
\text { uzorkovanja }\end{array}$ & $\begin{array}{c}\text { T }\left({ }^{\mathbf{0}} \mathbf{C}\right) \\
\text { vazduha }\end{array}$ & $\begin{array}{c}\text { T }\left({ }^{\mathbf{0}} \mathbf{C}\right) \\
\text { vode }\end{array}$ & Boja/miris & $\begin{array}{c}\text { El.provod. } \\
\boldsymbol{\mu S} / \mathbf{c m}\end{array}$ & $\mathbf{p H}$ & $\begin{array}{c}\mathbf{C u} \\
\left(\mathbf{m g} / \mathbf{d m}^{\mathbf{3}}\right)\end{array}$ & $\begin{array}{c}\mathbf{P b} \\
\left(\mathbf{m g} / \mathbf{d m}^{\mathbf{3}}\right)\end{array}$ & $\begin{array}{c}\mathbf{Z n} \\
\left(\mathbf{m g}_{\mathbf{d}} \mathbf{d m}\right)\end{array}$ \\
\hline Borska reka & 17.2 & 17.6 & Mutna/bez & 1472 & 5.00 & 16.9 & 0.23 & 2.9 \\
\hline
\end{tabular}

\begin{tabular}{|c|c|c|c|c|c|c|c|c|}
\hline $\begin{array}{c}\text { Mesto } \\
\text { uzorkovanja }\end{array}$ & $\begin{array}{c}\mathbf{C d} \\
\left(\mathbf{m g} / \mathbf{d m}^{\mathbf{3}}\right)\end{array}$ & $\begin{array}{c}\mathbf{N i} \\
\left(\mathbf{m g} / \mathbf{d m}^{\mathbf{3}}\right)\end{array}$ & $\begin{array}{c}\mathbf{C r} \\
\left(\mathbf{m g} / \mathbf{d m}^{\mathbf{3}}\right)\end{array}$ & $\begin{array}{c}\mathbf{S e} \\
\left(\mathbf{m g} / \mathbf{d m}^{\mathbf{3}}\right)\end{array}$ & $\begin{array}{c}\mathbf{A s} \\
\left(\mathbf{m g} / \mathbf{d m}^{\mathbf{3}}\right)\end{array}$ & $\begin{array}{c}\mathbf{F e}-t o t a l \\
\left(\mathbf{m g} / \mathbf{d m}^{\mathbf{3}}\right)\end{array}$ & $\begin{array}{c}\text { Sus. mater. } \\
\left(\mathbf{m g} / \mathbf{d m}^{\mathbf{3}}\right)\end{array}$ & $\begin{array}{c}\mathbf{S O}_{\mathbf{4}}{ }^{-2} \\
\left(\mathbf{m g} / \mathbf{d m}^{\mathbf{3}}\right)\end{array}$ \\
\hline Borska reka & $<0.1$ & 0.37 & $<0.1$ & $<0.2$ & $<0.1$ & 36.9 & 899.0 & 1204.3 \\
\hline
\end{tabular}

Takođe $\mathrm{u}$ istom periodu, dva puta mesečno je vršeno i uzimanje uzoraka vode iz piozometra izrađenog u priobalju
Borske reke. U tabelama 3 i $3 a$ su prikazani neki od rezultata analiza vode iz ovog piezometra. 
Tabela 3 i 3a. Rezultati fizičko hemijskih ispitivanja uzoraka podzemne vode

\begin{tabular}{|c|c|c|c|c|c|c|c|c|}
\hline $\begin{array}{c}\text { Mesto } \\
\text { uzorkovanja }\end{array}$ & $\begin{array}{c}\mathbf{T}\left(\mathbf{(}^{\mathbf{O} \mathbf{C})}\right. \\
\text { vazduha }\end{array}$ & $\begin{array}{c}\mathbf{T}\left(\mathbf{(}^{\mathbf{0}} \mathbf{C}\right) \\
\text { vode }\end{array}$ & Boja/miris & $\begin{array}{c}\text { El.provod. } \\
\mathbf{\mu S} / \mathbf{c m}\end{array}$ & $\mathbf{p H}$ & $\begin{array}{c}\mathbf{C u} \\
\left(\mathbf{m g} / \mathbf{d m}^{\mathbf{3}}\right)\end{array}$ & $\begin{array}{c}\mathbf{P b} \\
\left(\mathbf{m g} / \mathbf{d m}^{\mathbf{3}}\right)\end{array}$ & $\begin{array}{c}\mathbf{Z n} \\
\left(\mathbf{m g} / \mathbf{d m}^{\mathbf{3}}\right)\end{array}$ \\
\hline Borska reka & 20 & 16.0 & Mutna/bez & 1777 & 5.21 & 5.1 & $<0.1$ & 2.9 \\
\hline
\end{tabular}

\begin{tabular}{|c|c|c|c|c|c|c|c|c|}
\hline $\begin{array}{c}\text { Mesto } \\
\text { uzorkovanja }\end{array}$ & $\begin{array}{c}\mathbf{C d} \\
\left(\mathbf{m g} / \mathbf{d m}^{\mathbf{3}}\right)\end{array}$ & $\begin{array}{c}\mathbf{N i} \\
\left(\mathbf{m g} / \mathbf{d m}^{\mathbf{3}}\right)\end{array}$ & $\begin{array}{c}\mathbf{C r} \\
\left(\mathbf{m g} / \mathbf{d m}^{\mathbf{3}}\right)\end{array}$ & $\begin{array}{c}\text { Se } \\
\left(\mathbf{m g} / \mathbf{d m}^{\mathbf{3}}\right)\end{array}$ & $\begin{array}{c}\mathbf{A s} \\
\left(\mathbf{m g} / \mathbf{d m}^{\mathbf{3}}\right)\end{array}$ & $\begin{array}{c}\mathbf{F e}-t o t a l \\
\left(\mathbf{m g} / \mathbf{d m}^{\mathbf{3}}\right)\end{array}$ & $\begin{array}{c}\text { Sus. mater. } \\
\left(\mathbf{m g} / \mathbf{d m}^{\mathbf{3}}\right)\end{array}$ & $\begin{array}{c}\mathbf{S O}_{\mathbf{4}} \mathbf{- 2}^{-2} \\
\left(\mathbf{m g} / \mathbf{d m}^{\mathbf{3}}\right)\end{array}$ \\
\hline Borska reka & $<0.1$ & 0.37 & $<0.1$ & $<0.2$ & $<0.1$ & 36.9 & 899.0 & 1204.3 \\
\hline
\end{tabular}

\section{ZAKLJUČAK}

U republici Srbiji, zaštita površinskih voda vrši se zakonskom regulativom, koja se zasniva na klasifikaciji voda na četiri klase prema nivou zagađenosti i upotrebi. Granične vrednosti elemenata date su u Službenom listu Republike Srbije br. 31/82 (parametrihemijskog kvaliteta).

$\mathrm{Na}$ osnovu ovog pravilnika površinske vode Borske reke po svom sastavu ne mogu da se svrstaju ni u IV klasu i spadaju u vode sa visokim sadržajem y potencijalno toksičnih elemenata (teški metali) prema nivou maksimalno dozvoljene koncentracije.

Ovakvo zagađenje ima izuzetno veliki negativan uticaj po zdravlje stanovnika sela Slatina koje se nalazi nizvodno od grada Bora i industrijskih kompleksa koj zagađuju Borsku reku, a koja protiče kroz samo selo.

Povoljno kretanje cene bakra na svetskom tržištu je uticalo i na proizvodnju bakra u Boru koja raste iz godine u godinu. Takođe sa povećanjem proizvodnje raste i nivo zagađenja u Borskoj reci.

\section{LITERATURA}

[1] K. Marika, R. Cupac, B. Vulikic, D. Simić, A. Kodzoman, A. Nacev, E. Jusufi, I. Derguti, S. Dragojević, S. Marstijepović, 2007-2010, Program Western Balkans Environmental Programme Final Report (UNDP).

[2] Group of authors, 2002, Assessment of Environmental Monitoring Capacities in Bor, (UNEP).

[3] Group of authors, 2007, RTB Bor Tailing Ponds Remediation Study Final Report.

[4] Group of authors, 2011., Feasibility Study for Remediation the Ground and Surface Water in the Bor Mine.

[5] INTREAT - FP6 The Project Funded by the EU - Integrated Waste Treatment in Order to Prevent Pollution of the Danube River, MMI is a Partner in the Project, a Project Implemented from July 2004 to August 2007.

[6] Uredba Republike Srbije o deponijama (,Službeni gl. RS“ br. 92/2010).

[7] D. Ignjatović, L. Djurdjevac Ignjatović, M. Ljubojev: Uticaj pomeranja flotacijske brane 2 na trasu budućeg kolektora kriveljske reke, testiran pomoću softvera Phase2 v8.0, Mining and Metallurgy Engineering Bor, 4/2013, str. 23-28.

[8] Lj. Obradović, J. Stanković, M. Bugarin, Deponovanje opasnog otpada iz rudarstva - aktuelna zakonska regulativa EU i Srbije, Mining and Metallurgy Engineering Bor, 3/2013, str. 119-124. 